\title{
Broadband Acoustic Source Processing in a Noisy Shallow Ocean Environment
}

J. V. Candy, Lawrence Livermore National Laboratory

E. J. Sullivan, Naval Undersea Warfare Center

This paper was prepared for submittal to the

Oceans 96 Marine Technology Society (MTS)/IEEE

Ft. Lauderdale, Florida

September 23-26, 1996

July 18, 1996
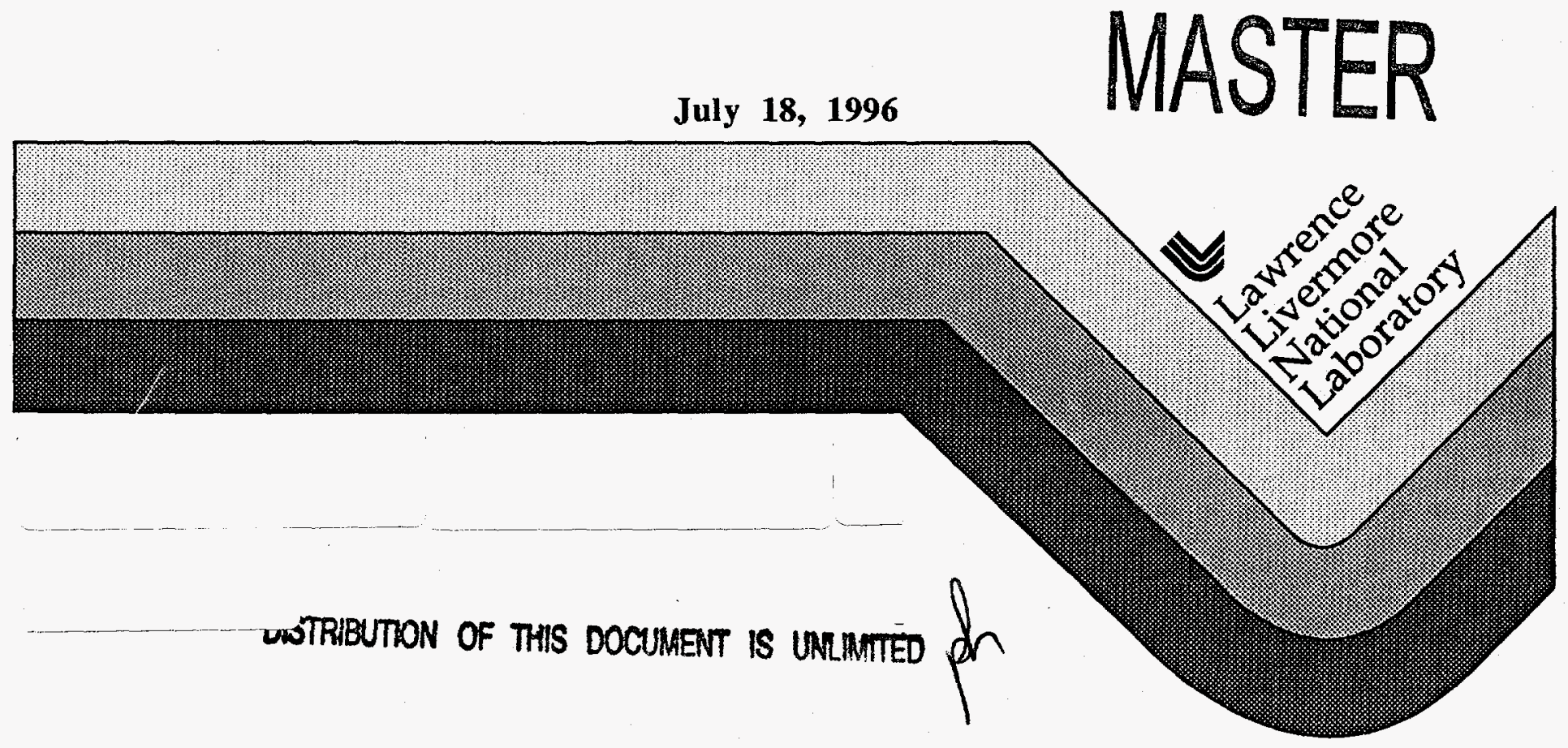


\section{DISCLAIMER}

This document was prepared as an account of work sponsored by an agency of the United States Government. Neither the United States Government nor the University of California nor any of their employees, makes any warranty, express or implied, or assumes any legal liability or responsibility for the accuracy, completeness, or usefulness of any information, apparatus, product, or process disclosed, or represents that its use would not infringe privately owned rights. Referenœ herein to any specific commercial product, process, or service by trade name, trademark, manufacturer, or otherwise, does not necessarily constitute or imply its endorsement, recommendation, or favoring by the United States Government or the University of California. The views and opinions of authors expressed herein do not necessarily state or reflect those of the United States Government or the University of California, and shall not be used for advertising or product endorsement purposes.

\section{DISCLAIMER}

This report was prepared as an account of work sponsored by an agency of the United States Government. Neither the United States Government nor any agency thereof, nor any of their employees, makes any warranty, express or implied, or assumes any legal liability or responsibility for the accuracy, completeness, or usefulness of any information, apparatus, product, or process disclosed, or represents that its use would not infringe privately owned rights. Reference herein to any specific commercial product, process, or service by trade name, trademark, manufacturer, or otherwise does not necessarily constitute or imply its endorsement, recommendation, or favoring by the United States Government or any agency thereof. The views and opinions of authors expressed herein do not necessarily state or reflect those of the United States Government or any agency thereof. 


\section{DISCLAIMER}

Portions of this document may be illegible in electronic image products. Images are produced from the best available original document. 


\section{Broadband Acoustic Source Processing in a Noisy Shallow Ocean Environment}

\author{
J.V. Candy \\ Lawrence Livermore National Laboratory \\ Livermore, CA 94550
}

\author{
E.J. Sullivan \\ Naval Undersea Warfare Center \\ Newport, RI 02841
}

\begin{abstract}
Acoustic sources found in the ocean environment are spatially complex and broadband, complicating the analysis of received acoustic data considerably. A model-based approach is developed for a broadband source in a shallow ocean environment characterized by a normalmode propagation model. Here we develop the "optimal" Bayesian solution to the broadband pressure-field enhancement and modal function extraction problem.
\end{abstract}

\section{INTRODUCTION}

Acoustic sources found in the hostile ocean environment are complex both spatially and temporally being broadband rather than narrowband. When propagating in the shallow ocean these source characteristics complicate the analysis of received acoustic data considerably-especially in littoral regions providing an important challenge for signal processing. It is this broadband or transient source problem that leads us to a model-based signal enhancement solution.

The localization of transient sources has long been a problem with keen interest in the ocean acoustic community primarily because most targets are broadband. Initial efforts began with a temporal matched-filter approach [1-4]. Here the assumed source location was used to provide a replica employed to match to the measured data. An alternative to the temporal matched-filter evolved as a natural extension to matched-field/matched mode processing which entails the "matching" of a predicted to a measured pressure-field under the assumed source location. After an exhaustive search, the location selected was that which provided a maximum value in the so-called ambiguity surface [5-10]. The uncertainty of the ocean medium motivates the use of stochastic models to capture the random nature of the phenomena ranging from ambient noise and scattering to distant shipping [11-12]. Therefore, processors that do not take these effects into account are doomed to large estimation errors. When contemplating the broadband problem, it is quite natural to develop temporal techniques especially if the underlying model is the full wave equation; however, if we assume a normal-mode propagation model then it seems more natural to: (1) filter the broadband receiver outputs into narrow bands;
(2) process each band with a devoted processor; and then (3) combine the narrowband results $[5,7,13]$ to create a broadband solution. One apparent advantage to this approach is to utilize narrowband signal processing techniques thereby providing some noise rejection and intermediate enhancement for the next stage. This is the approach we take in this paper to construct our broadband model-based processor (MBP).

Employing a vertical array of hydrophone sensors, the enhancement of noisy broadband acoustic pressurefield measurements using a multichannel model-based technique is discussed. Here a model-based approach is developed for the broadband source using a normalmode propagation model. Propagation theory predicts that a different modal structure evolves for each spectral line; therefore, it is not surprising that the multichannel model-based solution to this problem results in a scheme that requires a "bank" of processors-each employing its own underlying modal structure for the narrow frequency band that it operates over. The model-based solution using state-space forward propagators was developed using first principles where it was shown that each processor is decoupled in modal space and recombined in the measurement space to provide enhanced estimates [13].

We provide a brief discussion of the broadband problem in Sec. II and state the underlying state-space model structures discussed previously in [13]. Next in Sec. III we develop the broadband model-based processor showing that it is in fact the "optimal" Bayesian solution to the signal enhancement problem. We summarize our results in the final section and discuss future work.

\section{BROADBAND STATE PROPAGATORS}

In this section we briefly review the structure of the broadband propagator employed in a model-based scheme [13]. It is well-known in ocean acoustics that the pressure-field solution to the Helmholtz equation under the appropriate assumptions can be expressed as the sum of normal modes [14]. This modal representation has been extended to include a broadband source, $s(t)$, with corresponding spectrum, $S_{s}(\omega)$ [7-10]. In this case, the ocean medium is specified by its Green's function (impulse response) which can be expressed in terms of 
the inherent normal modes spanning the water column and therefore the resulting pressure-field in the temporal frequency domain is given by

$$
p(r, z, \omega)=\mathcal{G}(r, z, \omega) S(\omega)
$$

which corresponds to a convolution of $\mathcal{G}(r, z, t)$ and $s(t)$ in the time domain.

Suppose we decompose the continuous source spectrum into a discrete spectrum using a discrete Fourier series representation, then it follows that at the $q^{\text {th }}$ source frequency, we have

$$
p\left(r, z, \omega_{q}\right)=\mathcal{G}(r, z, \omega) S_{s}\left(\omega_{q}\right)
$$

where $S\left(\omega_{q}\right)$ can be interpreted as a single narrowband impulse at $\omega_{q}$ with amplitude or Fourier coefficient, $a_{q}=\Delta \omega\left|S\left(\omega_{q}\right)\right|$. Suppose that the broadband source is assumed to be bandlimited $\left(\omega_{1} \leq \omega \leq \omega_{0}\right)$, then the normal-mode solution to the Helmholtz equation for the broadband source problem can be decomposed into a series of narrowband solutions, that is,

$$
p\left(r, z, \omega_{q}\right)=\sum_{m=1}^{M_{q}} a_{q} \mathcal{H}_{0}\left(\kappa_{r}(m, q) r\right) \phi_{m}\left(z_{s}, \omega_{q}\right) \phi_{m}\left(z, \omega_{q}\right)
$$

with corresponding dispersion relation satisfying

$\kappa_{r}^{2}(m, q)=\frac{\omega_{q}^{2}}{c^{2}(z)}-\kappa_{z}^{2}(m, q), m=1, \ldots, M_{q} ; q=1, \ldots, Q$

where $p$ is the acoustic pressure-field; $a_{q}$ is the source amplitude; $\mathcal{H}_{0}$ is the zeroth order Hankel function; $\phi_{m}$ is the $m^{\text {th }}$ modal function evaluated at $z$ and source depth $z_{s} ; \kappa_{r}(m, q)$ is the horizontal wave number associated with the $m^{\text {th }}$ mode; $\kappa_{z}$ is the vertical wave number; $c$ is the depth-dependent sound speed profile; $\omega_{q}$ is the narrowband temporal source frequency; and $r$ is the horizontal range.

We further assume an L-element vertical sensor array, then $z \rightarrow z_{\ell}, \ell=1, \cdots, L$ and therefore, the pressurefield at the array for the $q^{\text {th }}$ temporal frequency becomes

$$
p\left(r, z_{\ell}, \omega_{q}\right)=\sum_{m=1}^{M_{q}} \beta_{m}\left(r, z_{\ell}, \omega_{q}\right) \phi_{m}\left(z_{\ell_{3}} \omega_{q}\right)
$$

where $\beta_{m}\left(r, z_{\ell}, \omega_{q}\right) \equiv a_{q} \mathcal{H}_{o}\left(\kappa_{r}(m, q) r\right) \phi_{m}\left(z_{s}, \omega_{q}\right)$ is the $m^{t h}$-modal coefficient at the $q^{t h}$ temporal frequency.

The next question of the broadband problem is, "how can this approach be used in a MBP solution?" It is well-known that the state-space propagators for the narrowband pressure-field can easily be obtained from a "depth only" model [15]. This model is valid for a single temporal frequency, and we would like to extend it to the broadband problem considering a set of temporal frequencies and associated amplitudes. We see from Ref. 13 that not only do the temporal frequencies $\left(\omega_{q}\right)$ change, but so do the number of modes $\left(M_{q}\right)$ spanning the water column at each frequency as well as the corresponding wave numbers $\left(\kappa_{r}(m, z)\right)$. Therefore, these variations imply that the state-space processor for the broadband case must also reflect these changes.

Including all of the temporal frequencies, $\left\{\omega_{q}\right\}, q=$ $1, \cdots, Q$, the overall broadband state-space propagator evolves as

$$
\begin{aligned}
& \frac{d}{d z} \Phi(z, \omega)=\mathbf{A}(z, \omega) \Phi(z, \omega) \\
& \mathrm{p}\left(z_{\ell}, \omega\right)=\mathbf{C}^{T}\left(r_{s}, z_{s}, \omega\right) \Phi\left(z_{\ell}, \omega\right)
\end{aligned}
$$

where $\boldsymbol{\Phi}(z, \omega) \in \mathbf{R}^{2 M \times 1}, \mathbf{A}(z, \omega) \in \mathbf{C}^{2 M \times 2 M}, \mathbf{p} \in$ $C^{Q \times 1}, C^{T} \in R^{Q \times 2 M}$ with $M \equiv \sum_{q=1}^{Q} M_{q}$. Note we use the parameter " $\omega$ " to signify the entire set of discrete temporal frequencies, $\left\{\omega_{q}\right\}, q=1, \cdots, Q$.

The internal structure of this overall processor admits the following decomposition:

$$
\begin{aligned}
& \frac{d}{d z} \Phi(z, \omega)=\left[\begin{array}{ccc}
\mathbf{A}\left(z, \omega_{1}\right) & \cdots & 0 \\
& \ddots & \\
0 & \cdots & \mathbf{A}\left(z, \omega_{Q}\right)
\end{array}\right]\left[\begin{array}{c}
\Phi\left(z, \omega_{1}\right) \\
\vdots \\
\Phi\left(z, \omega_{Q}\right)
\end{array}\right], \\
& \underset{\mathbf{R}^{2 M_{q} \times 2 M_{q} \text { for }}}{\mathbf{A}\left(z, \omega_{q}\right)}=\operatorname{diag}\left[\mathbf{A}_{1}\left(z, \omega_{q}\right) \cdots \mathbf{A}_{M_{q}}\left(z, \omega_{q}\right)\right] \in
\end{aligned}
$$$$
\mathbf{A}_{m}\left(z, \omega_{q}\right)=\left[\begin{array}{cc}
0 & 1 \\
-\kappa_{z}^{2}(m, q) & 0
\end{array}\right], \quad m=1, \cdots, M_{q}
$$

with the pressure-field sensor measurement model given by

$$
\mathrm{p}\left(z_{\ell}, \omega\right)=\left[\begin{array}{ccc}
C^{T}\left(z_{\ell}, \omega_{1}\right) & \cdots & 0 \\
& \ddots & \\
0 & \cdots & C^{T}\left(z_{\ell}, \omega_{Q}\right)
\end{array}\right] \Phi\left(z_{\ell}, \omega\right)
$$

As before in previous work [15] this deterministic model can be extended to a stochastic Gauss-Markov representation given by

$$
\begin{aligned}
& \frac{d}{d z} \Phi(z, \omega)=\mathbf{A}(z, \omega) \Phi(z, \omega)+\mathbf{w}(z, \omega) \\
& p\left(z_{\ell}, \omega\right)=\mathbf{C}^{T}(z, \omega) \Phi\left(z_{\ell}, \omega\right)+v(z, \omega)
\end{aligned}
$$

where $w, v$ are additive, zero-mean gaussian noise sources with respective spectral covariance matrices $\mathbf{R}_{w w}(z, \omega)$, and $\mathbf{R}_{v v}(z, \omega)$. Next we develop the broadband model-based processor.

\section{BROADBAND PROCESSING}

The broadband pressure-field can be characterized by the function, $p(z, \omega)$. If we assume that the field is measured by a vertical array, then at each sensor the acquired time series is given by $p\left(z_{\ell}, t\right)$. Clearly, this sensor measurement contains all of the information about 
the source, both temporally and spatially, but due to the complexity of this received data coupled with the noise, the required signal processing is quite a challenging problem. If we take the Fourier transform in the temporal domain, then the broadband source can be thought of as being viewed through a bank of narrowband temporal filters, that is, the broadband pressure-field surface or image is then given by: $p(\mathrm{z}, \omega) \rightarrow p\left(z_{\ell}, \omega_{q}\right)$; for $\ell=1, \cdots, L$, and $q=1, \cdots, Q$. Once this surface is constructed, it is possible to infer other useful information about the dynamics of the ocean as well as the source. If we assume further that we have a shallow water environment characterized by trapped wave propagation, then we may represent the underlying Green's function or channel impulse response in terms of a normal-mode model. In this case it may also be of interest to observe the surface created by the various broadband modal functions, that is, as a function of temporal frequency, $\phi_{m}\left(z_{\ell}, \omega_{q}\right)$; for $m=1, \cdots, M_{q}$. It is clear that as we decompose this complex temporal pressure-field measurement into these narrow frequency bands, each band will contain oceanographical and source information. Thus, the problem that we address first here, is that of receiving a set of temporal noisy broadband pressure-field measurements and developing the enhancement necessary to construct the surfaces created by both the broadband pressure-field and modal functions.

In order to obtain the optimal (minimum error variance) estimator, we cast our problem into a probabilistic framework under these Gauss-Markov assumptions; therefore, our sequence of pressure-field measurements at each sensor are Fourier transformed to yield a discrete set of frequencies $\left\{\omega_{q}\right\}, q=1, \cdots, Q$ and the set of broadband sensor measurements, $\left\{\mathrm{p}\left(z_{\ell}, \omega\right)\right\}, \ell=$ $1, \cdots, L ; \mathrm{p} \in C^{Q \times 1}$. Note that the window length of the Fourier transform is determined by the temporal correlation time of the measurement (source) to assure the independence of the frequency samples. For our pressurefield/modal function estimation problem, we define the underlying broadband pressure-field/modal function enhancement problem as:

GIVEN a set of noisy broadband pressure-field measurements, $\left\{\mathrm{p}\left(z_{\ell}, \omega\right)\right\}, \ell=1, \cdots, L$ and the underlying Gauss-Markov model (previous section), FIND the best (minimum error variance) estimate of the broadband modal functions, $\hat{\Phi}\left(z_{\ell}, \omega\right)$, and pressure-field, $\hat{\mathrm{p}}\left(z_{\ell}, \omega\right)$.

The minimum variance solution to this problem can be obtained by the maximizing the a posteriori density as follows. Define the set of broadband pressure-field measurements as: $P_{L} \equiv\left\{\mathrm{p}\left(z_{1}, \omega\right), \cdots, \mathrm{p}\left(z_{L}, \omega\right)\right\} ; \mathrm{p} \in$ $C^{Q \times 1}$, then the maximum a-posteriori (MAP) estimator of the modal functions must maximize the posterior density given by

$$
\operatorname{Pr}\left(\Phi\left(z_{\ell}, \omega\right) \mid P_{\ell}\right)=\frac{\operatorname{Pr}\left(\Phi\left(z_{\ell}, \omega\right), P_{\ell}\right)}{\operatorname{Pr}\left(P_{\ell}\right)}
$$

but from Bayes' rule we have that

$$
\operatorname{Pr}\left(\Phi\left(z_{\ell}, \omega\right) \mid P_{\ell}\right)=\frac{\operatorname{Pr}\left(\mathrm{p}\left(z_{\ell}, \omega\right) \mid \Phi\left(z_{\ell, \omega}, P_{\ell-1}\right)\right.}{\operatorname{Pr}\left(\mathbf{p}\left(z_{\ell}, \omega\right) \mid P_{\ell-1}\right)}
$$

$$
\times \frac{\operatorname{Pr}\left(\Phi \left(x_{\left.\ell, \omega), P_{\ell-1}\right)}\right.\right.}{\operatorname{Pr}\left(P_{\ell-1}\right)}
$$

Now expanding the second term in the numerator again using Bayes' rule, we have

$$
\operatorname{Pr}\left(\Phi\left(z_{\ell}, \omega\right), P_{\ell-1}\right)=\operatorname{Pr}\left(\Phi\left(z_{\ell-1}, \omega\right) \mid P_{\ell-1}\right) \operatorname{Pr}\left(P_{\ell-1}\right) .
$$

Substituting this relation in the previous equation and cancelling like terms we obtain the required expression for the posteriori density

$$
\begin{gathered}
\operatorname{Pr}\left(\Phi\left(z_{\ell}, \omega\right) \mid P_{\ell}\right)=\frac{\operatorname{Pr}\left(\mathrm{p}\left(z_{\ell}, \omega\right) \mid \Phi\left(z_{\ell}, \omega\right), P_{\ell-1}\right)}{\operatorname{Pr}\left(\mathbf{P}\left(z_{\ell}, \omega\right) \mid P_{\ell-1}\right)} \\
\times \operatorname{Pr}\left(\Phi\left(z_{\ell}, \omega\right) \mid P_{\ell-1}\right) .
\end{gathered}
$$

Under the Gauss-Markov assumptions of the previous section, we have that

$$
\begin{gathered}
\operatorname{Pr}\left(\mathrm{p}\left(z_{\ell}, \omega\right) \mid P_{\ell-1}\right) \sim \mathcal{N}\left(\mathbf{C}\left(r_{s}, z_{s}, \omega\right) \hat{\Phi}\left(z_{\ell \mid \ell-1}, \omega\right),\right. \\
\left.\mathrm{C}\left(r_{s}, z_{s}, \omega\right) \tilde{\mathbf{P}}\left(z_{\ell \mid \ell-1}, \omega\right) \mathbf{C}^{T}\left(r_{s}, z_{s}, \omega\right)+\mathbf{R}_{v v}\left(z_{\ell-1}, \omega\right)\right) \\
\operatorname{Pr}\left(\mathbf{p}\left(z_{\ell}, \omega\right) \mid \Phi\left(z_{\ell}, \omega\right), P_{\ell-1}\right) \sim \mathcal{N}\left(\mathbf{C}\left(r_{s}, z_{s}, \omega\right) \Phi\left(z_{\ell}, \omega\right),\right. \\
\left.\mathbf{R}_{v v}\left(z_{\ell-1}, \omega\right)\right)
\end{gathered}
$$

and

$$
\operatorname{Pr}\left(\Phi\left(z_{\ell}, \omega\right) \mid P_{\ell-1}\right) \sim \mathcal{N}\left(\hat{\Phi}\left(z_{\ell \mid \ell-1}, \omega\right), \tilde{\mathbf{P}}\left(z_{\ell \mid \ell-1}, \omega\right)\right) .
$$

where

$$
\begin{gathered}
\tilde{\mathbf{P}}\left(z_{\ell \mid \ell-1}, \omega\right)=\mathbf{A}\left(z_{\ell-1}, \omega\right) \tilde{\mathbf{P}}\left(z_{\ell-1 \mid \ell-1}, \omega\right) \mathbf{A}^{T}\left(z_{\ell-1}, \omega\right) \\
+\mathrm{R}_{w w}\left(z_{\ell-1}, \omega\right)
\end{gathered}
$$

Here the notation $\hat{\Phi}\left(z_{\ell \mid \ell-1}, \omega\right) \equiv E\left\{\Phi\left(z_{\ell}, \omega\right) \mid P_{\ell-1}\right\}$ is the conditional mean, that is, the "best" (minimum variance) estimate at depth $z_{\ell}$ based on the previous sensor measurement up to the depth $z_{\ell-1}$. The symbol $\sim$ means "distributed as" and $\mathcal{N}(m, v)$ is a gaussian distribution of mean $m$ and variance $v$.

Now substituting these distributions into the aposteriori density and performing the necessary manipulations (see Ref. 16 for details), we obtain

$$
\begin{gathered}
\operatorname{Pr}\left(\Phi\left(z_{\ell}, \omega\right) \mid P_{\ell-1}\right)=\mathcal{C}_{\ell} \exp \left\{-\frac{1}{2}\left(\mathbf{v}\left(z_{\ell}, \omega\right) \mathbf{R}_{v v}^{-1}\left(z_{\ell}, \omega\right)\right.\right. \\
\mathbf{v}^{T}\left(z_{\ell}, \omega\right)+\tilde{\Phi}^{T}\left(z_{\ell \mid \ell-1}, \omega\right) \tilde{\mathbf{P}}^{-1}\left(z_{\ell \mid \ell-1}, \omega\right) \tilde{\Phi}\left(z_{\ell \mid \ell-1}, \omega\right) \\
\left.\left.-\epsilon^{T}\left(z_{\ell}, \omega\right) \mathbf{R}_{\epsilon \epsilon}^{-1}\left(z_{\ell}, \omega\right) \epsilon\left(z_{\ell}, \omega\right)\right)\right\}
\end{gathered}
$$


where the broadband modal estimation error is defined by

$$
\tilde{\Phi}\left(z_{\ell \mid \ell-1}, \omega\right) \equiv \Phi\left(z_{\ell}, \omega\right)-\hat{\Phi}\left(z_{\ell \mid \ell-1}, \omega\right),
$$

and the broadband innovation is

$$
\epsilon\left(z_{\ell}, \omega\right) \equiv \mathrm{p}\left(z_{\ell,} \omega\right)-\hat{\mathrm{p}}\left(z_{\ell \mid \ell-1}, \omega\right),
$$

with the enhanced (MAP estimated) broadband measurement defined by

$$
\hat{\mathbf{p}}\left(z_{\ell}, \omega\right) \equiv \mathbf{C}\left(r_{s}, z_{s}, \omega\right) \hat{\mathbf{\Phi}}\left(z_{\ell \mid \ell-1}, \omega\right),
$$

and the constant $\mathcal{C}_{\boldsymbol{\ell}}$ with respective spectral covariance matrices, $\tilde{\mathbf{P}}\left(z_{\ell-1 \mid \ell-1}, \omega\right)$ and $\mathbf{R}_{\epsilon \epsilon}\left(z_{\ell}, \omega\right)$. By maximizing the a-posteriori density or equivalently its logarithm [16], we have the MAP equation,

$$
\left.\frac{\partial}{\partial \Phi} \ln \operatorname{Pr}\left(\Phi\left(z_{\ell}, \omega\right) \mid P_{\ell-1}\right)\right|_{\Phi=\hat{\Phi}_{M A P}}=0 .
$$

Differentiating the posterior density and noting that $\hat{\Phi}\left(z_{\ell \ell-1}, \omega\right)$ and $\epsilon\left(z_{\ell}, \omega\right)$ are both functions of the data set, $P_{\ell-1}$, we obtain

$$
\begin{gathered}
\frac{\partial}{\partial \Phi} \ln \operatorname{Pr}\left(\Phi\left(z_{\ell}, \omega\right) \mid P_{\ell-1}\right)=\mathrm{C}\left(r_{s}, z_{s}, \omega\right) \mathrm{R}_{v v}^{-1}\left(z_{\ell}, \omega\right) \mathbf{v}\left(z_{\ell}, \omega\right) \\
-\tilde{\mathbf{P}}^{-1}\left(z_{\ell \mid \ell-1}, \omega\right) \tilde{\Phi}\left(z_{\ell \mid \ell-1}, \omega\right)=0
\end{gathered}
$$

Solving the MAP equation for $\Phi=\hat{\Phi}_{M A P}$ yields

$$
\begin{gathered}
\hat{\mathbf{\Phi}}_{M A P}\left(z_{\ell}, \omega\right)=\tilde{\mathbf{P}}\left(z_{\ell \mid \ell}, \omega\right)\left[\tilde{\mathbf{P}}^{-1}\left(z_{\ell \mid \ell-1}, \omega\right) \hat{\Phi}\left(z_{\ell \mid \ell-1}, \omega\right)\right. \\
\left.+\mathbf{C}^{T}\left(r_{s}, z_{s}, \omega\right) \mathbf{R}_{v v}^{-1}\left(z_{\ell}, \omega\right) \mathrm{p}\left(z_{\ell}, \omega\right)\right]
\end{gathered}
$$

where we have used the fact that

$$
\begin{gathered}
\tilde{\mathbf{P}}\left(z_{\ell \mid \ell}, \omega\right)=\left[\mathbf{C}\left(r_{s}, z_{s}, \omega\right) \mathbf{R}_{v v}^{-1}\left(z_{\ell}, \omega\right) \mathbf{C}^{T}\left(r_{s}, z_{s}, \omega\right)\right. \\
\left.+\tilde{\mathbf{P}}^{-1}\left(z_{\ell \mid \ell-1}, \omega\right)\right]^{-1}
\end{gathered}
$$

from the matrix inversion lemma. Using the expression for the Kalman gain, the optimal broadband MAP processor $\hat{\boldsymbol{\Phi}}_{\text {MAP }}\left(z_{\ell}, \omega\right)=\hat{\Phi}\left(z_{\ell \mid \ell}, \omega\right)$ evolves as (see Ref. 16 , pp. 80-81 for details)

$$
\hat{\Phi}\left(z_{\ell \mid \ell}, \omega\right)=\hat{\Phi}\left(z_{\ell \mid \ell-1}, \omega\right)+\mathbf{K}\left(z_{\ell}, \omega\right) \varepsilon\left(z_{\ell}, \omega\right)
$$

where $\hat{\Phi}\left(z, \omega_{q}\right) \in R^{2 M \times 1}, \mathbf{K}\left(z_{\ell}, \omega\right) \in \mathbf{C}^{2 M \times Q}$, and $\epsilon\left(z_{\ell}, \omega\right) \in \mathbf{C}^{Q \times 1}$. The overall structure of the estimator can be seen by expanding the gain matrix over the set of discrete frequencies for $\omega \rightarrow \omega_{q}$, one for each of the $Q$-columns to give

$$
\hat{\Phi}\left(z_{\ell \mid \ell}, \omega\right)=\hat{\Phi}\left(z_{\ell \mid \ell-1}, \omega\right)
$$

$$
+\left[\mathbf{K}\left(z_{\ell}, \omega_{1}\right) \cdots \mathbf{K}\left(z_{\ell}, \omega_{Q}\right)\right]\left[\begin{array}{c}
\epsilon\left(z_{\ell}, \omega_{1}\right) \\
\vdots \\
\epsilon\left(z_{\ell}, \omega_{Q}\right)
\end{array}\right],
$$

or

$$
\hat{\Phi}\left(z_{\ell \mid \ell}, \omega\right)=\hat{\Phi}\left(z_{\ell \mid \ell-1}, \omega\right)+\sum_{q=1}^{Q} \mathbf{K}\left(z_{\ell}, \omega_{q}\right) \epsilon\left(z_{\ell}, \omega_{q}\right) .
$$

where $\mathbf{K}\left(z_{\ell}, \omega_{q}\right) \in \mathbf{C}^{2 M \times 1}$, and $\epsilon\left(z_{\ell}, \omega_{q}\right) \in \mathbf{C}^{1 \times 1}$. Now let us rewrite this equation in a slightly different manner by expanding over the set of discrete frequencies and expressing the gain in terms of $2 M_{q} \times Q$ block rows

$\hat{\mathbf{\Phi}}\left(z_{\ell \mid \ell}, \omega\right)=\hat{\mathbf{\Phi}}\left(z_{\ell \mid \ell-1}, \omega\right)+\left[\begin{array}{c}\mathbf{K}_{1}^{T}\left(z_{\ell}, \omega\right) \\ \vdots \\ \mathbf{K}_{Q}^{T}\left(z_{\ell}, \omega\right)\end{array}\right] \epsilon\left(z_{\ell}, \omega\right)$.

If we further decompose each block row $K_{q}^{T}\left(z_{\ell}, \omega\right)$ into its individual column vectors defined by $\mathbf{K}_{q n}\left(z_{\ell}, \omega_{n}\right) \in$ $C^{2 M_{q} \times 1}$, that is,

$$
\mathbf{K}_{q}^{T}\left(z_{\ell}, \omega\right) \equiv\left[K_{q 1}\left(z_{\ell}, \omega_{1}\right) \cdots K_{q Q}\left(z_{\ell}, \omega_{Q}\right)\right]
$$

then we can obtain the narrowband recursion for the corrected estimate as

$$
\hat{\Phi}\left(z_{\ell \mid \ell}, \omega_{q}\right)=\hat{\Phi}\left(z_{\ell \mid \ell-1}, \omega_{q}\right)+\sum_{n=1}^{Q} \mathbf{K}_{q n}\left(z_{\ell}, \omega_{n}\right) \epsilon\left(z_{\ell}, \omega_{n}\right)
$$

with $\hat{\Phi}\left(z_{\ell \mid \ell}, \omega_{q}\right) \in \mathbf{C}^{2 M_{q} \times 1}$ the gain $\mathbf{K}_{q n}\left(z_{\ell}, \omega_{n}\right) \in$ $\mathbf{C}^{2 M_{q} \times 1}$, and $\epsilon\left(z_{\ell}, \omega_{n}\right) \in \mathbf{C}^{1 \times 1}$ showing how each narrowband frequency line $\omega_{q}$ can be combined to form the optimal MAP estimate. Note that the entire broadband pressure-field measurement is required at each sensor, since

$$
\epsilon\left(z_{\ell}, \omega\right)=\left[\begin{array}{c}
p\left(z_{\ell}, \omega_{1}\right) \\
\vdots \\
p\left(z_{\ell}, \omega_{Q}\right)
\end{array}\right]-\hat{\mathbf{p}}\left(z_{\ell \mid \ell-1}, \omega\right) .
$$

However, this relation suggests an efficient parallel, but suboptimal approach to implementing this broadband estimator might be achieved by constructing a "local" narrowband processor for each $\omega_{q}$ and then combining their outputs to obtain the final broadband estimate, that is,

$$
\hat{\Phi}\left(z_{\ell \mid \ell}, \omega_{q}\right)=\hat{\Phi}\left(z_{\ell \mid \ell-1}, \omega_{q}\right)+K_{q q}\left(z_{\ell}, \omega_{q}\right) \epsilon\left(z_{\ell}, \omega_{q}\right),
$$

where we have discarded the other $2 M_{q} \times 1$ submatrices, $\mathbf{K}_{q n}\left(z_{\ell}, \omega_{n}\right)=\mathbf{0} n \neq q$. To see this recall that the broadband Kalman gain is calculated from

$$
\mathbf{K}\left(z_{\ell}, \omega\right)=\tilde{\mathbf{P}}\left(z_{\ell \mid \ell-1}, \omega\right) \mathbf{C}^{T}\left(r_{s}, z_{s}, \omega\right) \mathbf{R}_{\epsilon \epsilon}^{-1}\left(z_{\ell}, \omega\right),
$$


and if we now assume the parallel (suboptimal) form, then the covariance is assumed block diagonal

$$
\tilde{\mathbf{P}}\left(z_{\ell \mid \ell-1}, \omega\right)=\operatorname{diag}\left[\tilde{\mathbf{P}}\left(z_{\ell \mid \ell-1}, \omega_{1}\right) \cdots \tilde{\mathbf{P}}\left(z_{\ell \mid \ell-1}, \omega_{Q}\right)\right]
$$

and thus performing the implied partitions and multiplying gives

$$
\mathbf{K}\left(z_{\ell}, \omega\right)=\left[\begin{array}{ccc}
\mathbf{K}_{11}\left(z_{\ell}, \omega_{1}\right) & & 0 \\
& \ddots & \\
0 & & \mathbf{K}_{Q Q}\left(z_{\ell}, \omega_{Q}\right)
\end{array}\right]
$$

where $\mathbf{K}_{q q}\left(z_{\ell}, \omega_{q}\right) \frac{\tilde{\mathbf{P}}\left(z_{\ell \mid \ell-1}, \omega_{q}\right) \mathbf{C}^{T}\left(r_{s}, z_{s}, \omega_{q}\right)}{\mathbf{R}_{\varepsilon \varepsilon}\left(z_{\left.\ell, \omega_{q}\right)}\right.}$.

It is interesting to return to the optimal solution and note some of its features. First from the physics of the normal-mode model (narrowband temporal frequency), it is necessary to develop a bank of optimal narrowband estimators at each bin, $\omega_{q}$ so that the broadband estimate can be reconstructed from the optimal set, $\left\{\hat{\mathrm{p}}\left(\omega_{q}\right)\right\}, q=1, \cdots, Q$ over the array. Note also that the broadband modal estimates are also constructed in a similar manner as $\left\{\hat{\Phi}\left(z_{\hat{k}}, \omega_{q}\right)\right\}, q=1, \cdots, Q$.

Thus, our implementation of the optimal algorithm can be processed as a bank of narrowband model-based predictors combined during the correction phase of the algorithm to create the optimal broadband MAP estimator. The estimation algorithm then proceeds for each $\left\{\omega_{q}\right\}, q=1, \therefore, Q$ as:

Prediction:

$$
\frac{d}{d z} \hat{\Phi}\left(z, \omega_{q}\right)=\mathbf{A}\left(z, \omega_{q}\right) \hat{\Phi}\left(z, \omega_{q}\right)
$$

\section{Innovation:}

$$
\epsilon\left(z_{\ell}, \omega_{q}\right)=p\left(z_{\ell}, \omega_{q}\right)-\mathbf{C}^{T}\left(r_{s}, z_{s}, \omega_{q}\right) \hat{\Phi}\left(z_{\ell \mid \ell-1}, \omega_{q}\right)
$$

Correction:

$$
\hat{\Phi}\left(z_{\ell \mid \ell}, \omega_{q}\right)=\hat{\Phi}\left(z_{\ell \mid \ell-1}, \omega_{q}\right)+\sum_{n=1}^{Q} K_{q n}\left(z_{\ell}, \omega_{n}\right) \epsilon\left(z_{\ell}, \omega_{n}\right)
$$

Gain:

$$
\mathbf{K}\left(z_{\ell}, \omega\right)=\tilde{\mathbf{P}}\left(z_{\ell \mid \ell-1}, \omega\right) \mathbf{C}^{T}\left(r_{s}, z_{s}, \omega_{q}\right) \mathbf{R}_{\epsilon \epsilon}^{-1}\left(z_{\ell}, \omega\right)
$$

This completes the description of the basic broadband MBP. To implement this processor we must first narrowband filter the sensor temporal signals using the the DFT approach, that is, if we expand this equation over all frequencies, then using the DFT each of the narrowband frequency samples can be separated and used as input to the appropriate MBP. The overall broadband pressure-field can be reconstructed by replacing the noisy measurement $p\left(z_{\ell}, \omega\right)$ with the estimated or enhanced pressure-field, $\hat{p}\left(z_{\ell \mid \ell-1}, \omega\right)$ predicted by the MBP. If we employ the processor to "enhance" the noisy pressure-field measurements, then the corresponding output of the MBP is simply

$$
\hat{\mathbf{p}}\left(z_{\ell}, \omega\right)=\mathbf{C}\left(r_{s}, z_{s}, \omega\right) \hat{\Phi}\left(z_{\ell} \mid z_{\ell-1}, \omega\right)
$$

with the extracted/enhanced modal function estimates given by $\hat{\Phi}\left(z_{\ell} \mid z_{\ell-1}, \omega\right)$ and of course, the corresponding innovations used to monitor the performance of the processor (see Ref. 13 for details). This completes the theoretical discussion of the development of the broadband model-based processor.

\section{DISCUSSION}

In this paper we have developed the optimal Bayesian solution to the broadband pressure-field enhancement and modal function extraction (enhancement) problem. Modeling a shallow ocean environment by a normalmode propagator, we developed the broadband modelbased solution. We showed how each of the corresponding temporal frequency bands lead to an underlying state-space structure which is eventually used in the development of a forward propagator for simulation and the optimal processor for enhancement, when the underlying processes are modeled as Gauss-Markov. It was also shown how a suboptimal MBP could be constructed by ignoring matrix terms in the corresponding optimal error covariance matrix leading to a block diagonal gain matrix and an inherent parallel processor.

It is also interesting to note that from this particular approach, an optimal broadband "localizer" should follow immediately. Recall from Ref. 17 that an adaptive form of the MBP, the model-based identifier (MBID) could be designed to extract the so-called range-depth functions (modal coefficients) defined by

$$
\theta\left(z_{\ell}\right)=\left[\begin{array}{lll|l}
\theta_{1}\left(z_{\ell}\right)|\cdots| & \theta_{M}\left(z_{\ell}\right)
\end{array}\right]^{T},
$$

along with the modal function estimates, $\left\{\hat{\theta}_{m 1}\left(z_{\ell}, \omega_{q}\right)\right\}$ leading to a nonlinear least-squares localizer. It seems clear, using this same framework, that the broadband model-based localizer (MBL) [18] would use an adaptive form of the processor developed in this paper to estimate broadband range-depth functions defined by

$$
\begin{gathered}
\theta\left(z_{\ell}, \omega\right)=\left[\theta_{1}\left(z_{\ell}, \omega_{q}\right)|\cdots| \theta_{m}\left(z_{\ell}, \omega_{q}\right)\right]^{T}, \\
m=1, \cdots, M_{q} ; \quad q=1, \cdots, Q .
\end{gathered}
$$

where the position estimates, $\left(r_{s}, z_{s}\right)$, are the solution to minimizing a nonlinear least-squares cost, but this is the subject of future work.

\section{ACKNOWIEDGMENT}

We especially would like to thank Dr. R. Doolitthe for motivating this effort and Cdr. M. Shipley and Dr. J. Tague of the Office of Naval Research (ONR) for their support as well as the continued support of Dr. T. Goldsberry and Dr. D. Johnson also of ONR. 


\section{References}

[1] A. Parvulescu. "Signal detection in a multipath medium by MESS processing," J. Acoust. Soc. Am., $29,223-228,1965$.

[2] C. S Clay. "Optimum time domain signal transmission and source localization in a waveguide," $J$. Acoust. Soc. Am., 81, 660-664, 1987.

[3] S. Li and C. S Clay. "Optimum time domain signal transmission and source localization in a waveguide: experiments in an ideal wedge waveguide," $J$. Acoust. Soc. Am., 82, 1409-1417, 1987.

[4] R. K. Brienzo and W. Hodgkiss, "Broadband matched-field processing". J. Acoust. Soc. Am., 94, 1409-1417, 1994.

[5] A. B. Baggeroer, W. A. Kuperman, and $H$. Schmidt, "Matched-field processing: source localization in correlated noise as an optimum parameter estimation problem," J. Acoust. Soc. Am, 83, (2), 571-587, 1988 .

[6] E. K. Westwood, "Broadband matched-field source localization," J. Acoust. Soc. Am, 91, (5), 2777$2789,1992$.

[7] T. C. Yang. "Broadband source localization and signature estimation," J. Acoust. Soc. Am, 93, (4), 1797-1806, 1993.

[8] I. T. Lu, H. Y. Chen, and P. Voltz. "A matchedmode processing technique for localizaing a transient source in the time domain," J. Acoust. Soc. $A m, 93,(3), 1365-1373,1993$.

[9] H. Y. Chen, and I. T. Lu. "Localization of a broadband source using a matched-mode procedure in the time-frequency domain," IEEE Oceanic Engr., 19, (2), 166-174, 1994.

[10] P. C. Mignerey and S. Finette, "Multichannel deconvolution of an acoustic transient in an ocean waveguide," $J$. Acoust. Soc. Am., 92, (1), 351-364, 1992.

[11] W. M. Carey and W. B. Moseley. "Space-time processing, environmental-acoustic effects." IEEE $J$. Oceanic Engr., 16, (3), 285-301, 1991.

[12] E .J. Sullivan and D. Middleton, "Estimation and detection issues in matched-field processing", IEEE Trans. Ocean Engr., 18, (3), 156-167, 1993

[13] J. V. Candy and E. J. Sullivan. "Model-based processing of broadband sources in noisy shallow ocean environments." J. Acoust. Soc. Am., 99, (4), Pt. 2; $2572,1996$.

[14] F. B. Jensen, W. A. Kuperman, M. B. Porter and H. Schmidt, Computational Ocean Acoustics. New York:AIP Press, 1994.

[15] J. V. Candy and E. J. Sullivan. "Ocean acoustic signal processing: a model-based approach." $J$. Acoust. Soc. Am.,92, (12), 3185-3201, 1992.
[16] J. V. Candy, Signal Processing: The Model-Based Approach. New York:McGraw-Hill, 1986.

[17] J. V. Candy and E. J. Sullivan. "Model-based identification an adaptive approach to ocean acoustic signal processing", IEEE Trans. Ocean Eng., 21, (6), 240-252, 1996 .

[18] J. V. Candy and E. J. Sullivan. "Ocean acoustic passive localization: a model-based approach." $J$. Acoust. Soc. Am., 96, (9), 3185-3201, 1995. 